\section{Pericarditis tuberculosa: una manifestación extrapulmonar infrecuente de TBC}

\author{
MANUEL JORQUERA-ROMÁN ${ }^{1,2, b}$ JAVIER ARAYA-CANCINO ${ }^{2, a}$, \\ JOSÉ ENRÍQUEZ-MONTENEGRO 2,a, JAVIER OBANDO-VALDÉS ${ }^{2, a}$, \\ FRANCO REYES-CORNEJO ${ }^{2, a}$, OWARD BELZAREZ GUTIÉRREZ ${ }^{1}$, \\ VALERIA MONASTERIO-ANGULO ${ }^{3}$, NICOLE JOFRE-TOBAR ${ }^{3}$, \\ JOSÉ BARRIENTOS VERDUGO ${ }^{4}$, FERNANDO RODRÍGUEZ ROJAS ${ }^{5}$
}

\section{Tuberculous pericarditis an infrequent extrapulmonary manifestation of TB}

Extrapulmonary tuberculosis (TB) contributes to $15 \%$ of total cases, representing a great diagnostic and therapeutic challenge. Pericardial involvement is present in 1 to $2 \%$ of TB patients and is considered an unusual presentation form of TB. We report a 67-year-old male presenting with fever and progressive dyspnea. A chest CAT scan showed a bilateral pleural effusion and an extensive pericardial effusion. An echocardiogram showed signs of tamponade. Therefore, an emergency pericardiectomy was performed. The pathological report of pericardial tissue showed caseating necrosis and its Koch culture was positive. The patient was treated with anti-tuberculous drugs with a favorable evolution.

(Rev Med Chile 2021; 149: 281-285)

Key words: Pericardial Effusion; Pericarditis, Constrictive; Tuberculosis; Tuberculosis, Cardiovascular.
'Servicio de Medicina Adulto, Hospital Regional de Talca. Talca, Chile.

${ }^{2}$ Escuela de Medicina, Universidad Católica del Maule. Talca, Chile. 3Unidad de Anatomía patológica, Hospital Regional de Talca. Talca, Chile.

${ }^{4}$ Equipo de Tórax. Servicio de Cirugía adulto. Hospital de Talca. Talca, Chile.

5Unidad de Cirugía cardiaca, Servicio de Cardiología y cirugía cardiaca. Hospital de Talca. Talca, Chile.

aLicenciado en Medicina.

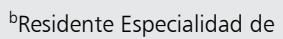
Medicina Interna.

Trabajo no recibió financiamiento. Los autores declaran no tener conflictos de interés.

Recibido el 10 de julio de 2020 aceptado el 15 de diciembre de 2020

Correspondencia a: Manuel Jorquera-Román Servicio de Medicina Adulto, Hospital Regional de Talca. 1 Norte \#1951, Talca, Chile. mjorquera@hospitaldetalca.cl
L a tuberculosis (TBC) es un problema de salud pública, durante el año 2018 diez millones de personas contrajeron esta enfermedad en el mundo ${ }^{1}$. A su vez, la TBC extrapulmonar contribuye al $15 \%$ del total de casos, representando un gran desafío en cuanto a su diagnóstico y tratamiento ${ }^{2}$, El compromiso pericárdico está presente en 1 a $2 \%$ de los pacientes con $\mathrm{TBC}^{3}$.

En Chile, la incidencia de tuberculosis fue de 15,6 casos por 100.000 habitantes para el año 2017, siendo $18,9 \%$ a casos de presentación extrapulmonar. ${ }^{4}$. En los últimos 20 años solo se han publicado 2 casos de TBC pericárdica nacionales ${ }^{5,6}$, constituyendo una forma de presentación poco estudiada en nuestro medio y un desafío diagnóstico. Actualmente con el aumento de la población migrante, en especial de regiones con mayor incidencia de TBC (Perú, Colombia, Venezuela y Haití) ${ }^{7}$, se debe considerar la TBC como factor etiológico de toda pericarditis con características sugerentes (filamentos de fibrina a la ecocardiografía y gran compromiso pericárdico), con el fin de instaurar un tratamiento precoz y minimizar la transmisión.

\section{Caso clínico}

Hombre de 67 años de edad, con antecedentes de hipertensión arterial, consultó por cuadro febril de $38,2^{\circ} \mathrm{C}$ de una semana de evolución, compromiso general, sudoración nocturna y disnea progresiva. Evaluado previamente en servicio de urgencia donde se inicia tratamiento antibiótico por cuadro compatible de neumonía bacteriana. Por persistencia de los síntomas, se decide hospitalizar en contexto de síndrome febril sin foco.

Se realiza estudio de laboratorio de ingreso que muestra creatinina $0,76 \mathrm{mg} / \mathrm{dL}$, albumina $2,8 \mathrm{~g} / \mathrm{dL}$, Ferritina $832 \mathrm{ng} / \mathrm{mL}$, leucocitos $3.320 \mathrm{~mm}^{3}$ (81\% segmentados), LDH 173 U/L, PCR 74,4 mg/L, serología para VIH, VHB, VHC y Sífilis no reactivos, 
además, se realizó estudio de inmunoglobulinas, complemento, ANA, ENA y anti-DNA, factor reumatoideo; todo dentro de limites normales.

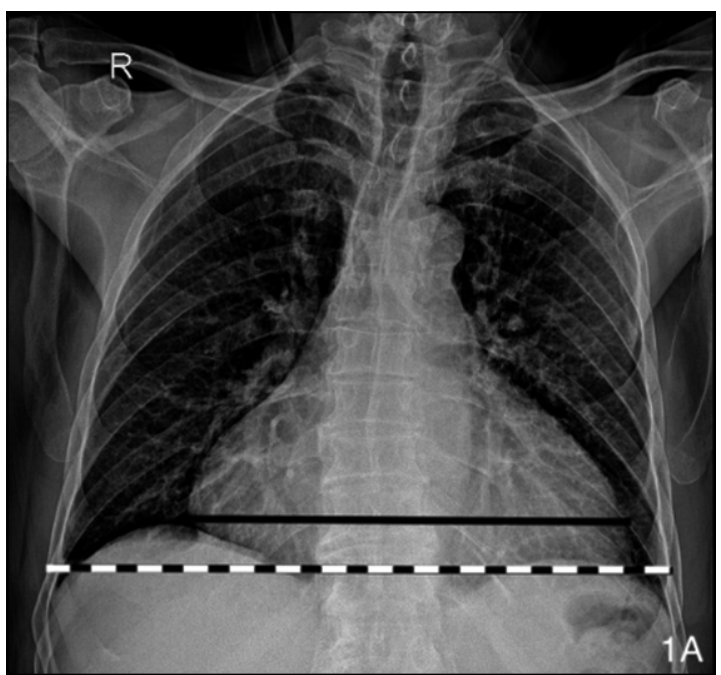

Figura 1A. Radiografía de tórax postero-anterior de ingreso; se observa aumento de la silueta cardiaca (línea continua) en comparación al diámetro del tórax (línea discontinua) en inspiración y un infiltrado peri hiliar bilateral, sin signos de condensación.
Se realiza radiografía de tórax (Figura 1A) que evidencia cardiomegalia e infiltrado pulmonar bilateral escaso. Se complementa con tomografía de Tórax que muestra leve derrame pleural bilateral, derrame pericardico extenso, e imágenes ganglionares calcificadas (Figura 1B).

Durante la hospitalización evoluciona con hipotensión, taquicardia y ruidos cardíacos apagados, se complementa estudio con ecocardiograma que informa derrame pericárdico severo con signos de taponamiento: colapso parcial del ventrículo derecho en diástole, hipocinesia global y Fracción de eyección de 40\%. Dado el contexto de taponamiento cardiaco se realiza ventana pericárdica de urgencia.

Se toma muestra de líquido pleural, líquido pericárdico y tejido pericárdico. El análisis citoquímico pleural informa aspecto turbio-amarillento, leucocitos $663 \mathrm{~mm}^{3}$, mononuclear $91 \%$, tinción de gram sin bacterias, glucosa $96 \mathrm{mg} / \mathrm{dL}$ y proteínas $3.833 \mathrm{mg} / \mathrm{dL}$. El citoquímico pericárdico: leucocitos $693 \mathrm{~mm}^{3}$, mononucleares $78 \%$, glucosa $32,9 \mathrm{mg} /$ dL y proteínas $4.554 \mathrm{mg} / \mathrm{dL}$. El análisis histológico (Figura 2) del tejido pericárdico informa un proceso inflamatorio crónico con células gigantes

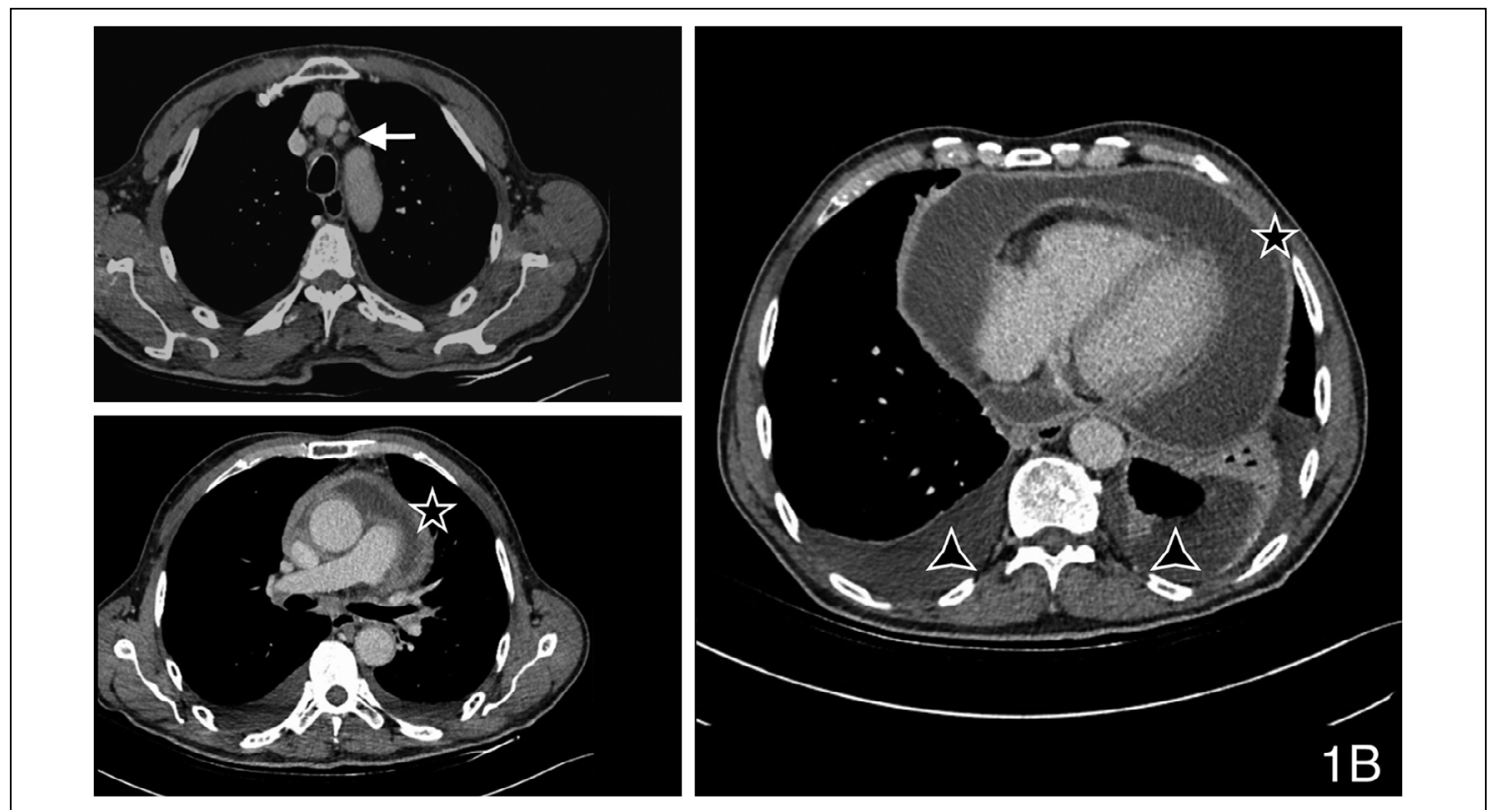

Figura 1B. Tomografía axial computada de tórax con contraste en fase venosa. Se observa de un derrame pleural (triángulo) en ambas bases pulmonares en leve cantidad a predominio derecho que concurre con discreta atelectasia pasiva basal bilateral. Se aprecian imágenes ganglionares (flecha) aumentadas de tamaño a nivel para-traqueal, pre-carinal, sub-carinal, pre-vascular y en la ventana aortocava. Corazón de tamaño y configuración conservado. Se aprecia acentuado derrame pericárdico (Estrella) alcanzando una profundidad de $35 \mathrm{~mm}$. 
multinucleadas y necrosis central caseificante. Se complementa estudio con la técnica reacción en cadena de polimerasa (PCR) en tiempo real con sondas especificas del Kit "Mycobacterium complex IS6110 repeat region" resultado positivo para Mycobacterium tuberculosis, al igual que el cultivo de Koch del tejido pericárdico, sensible a isoniacida y rifampicina, por lo que se decide iniciar tratamien- to a dosis fija combinada (Rifampicina-150 mg, Isoniacida-75 mg, Pirazinamida-400 mg y Etambutol-275 mg). A los 10 días es reevaluado con ecocardiograma que evidenció derrame pericárdico recidivante, por lo que se realiza nueva ventana pericárdica evolucionando de forma estable.

Se controla ecocardiograma tras 2 meses de inicio de terapia antifímica, reportando una frac-
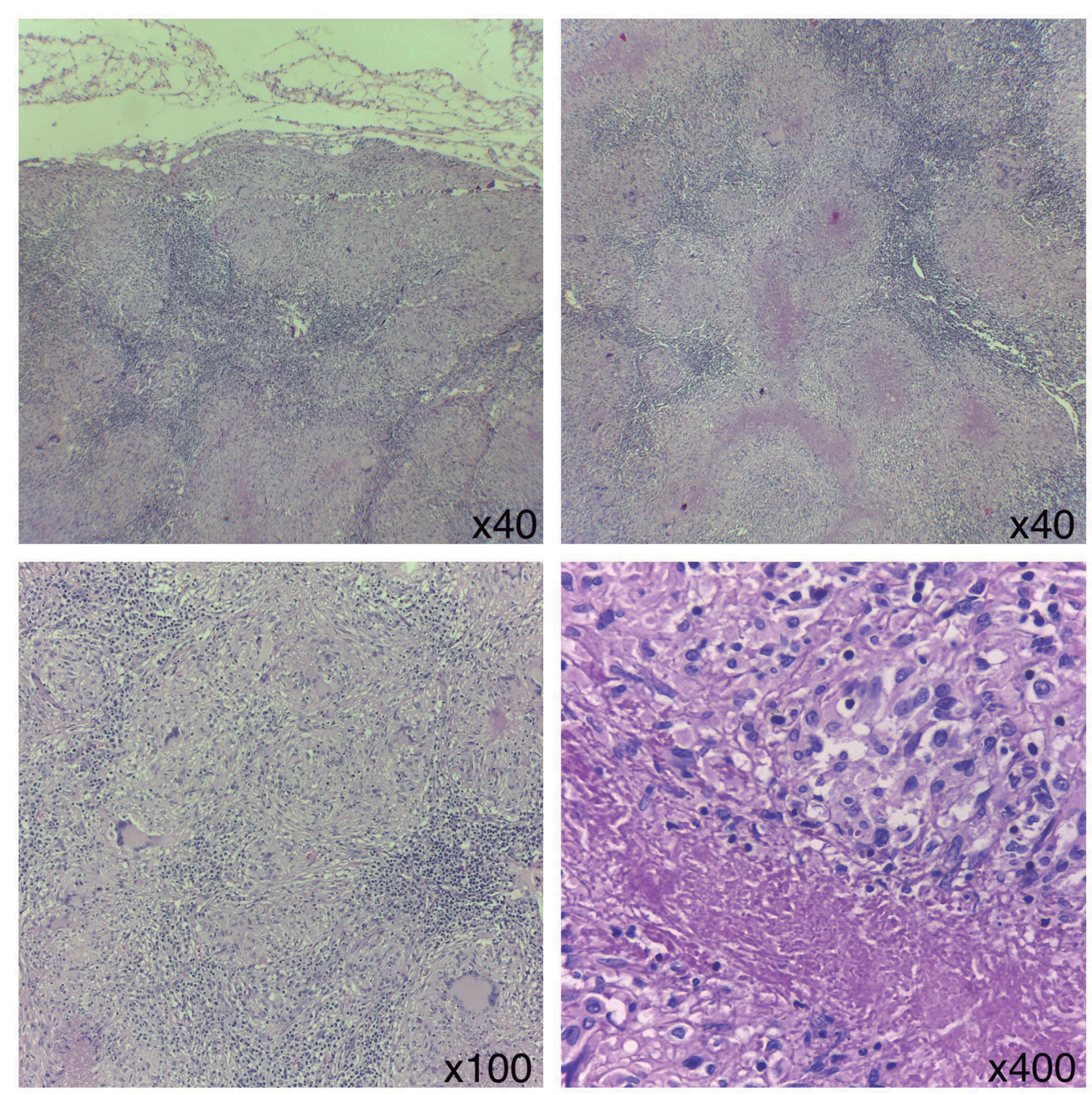

Figura 2. Tinción hematoxilina y eosina. Muestra constituida por tejido conectivo revestido parcialmente por mesotelio, de tipo pericardio engrosado dado por leve fibrosis con acentuado infiltrado inflamatorio crónico, constituido por formaciones nodulillares confluentes, conformado predominantemente por macrófagos epiteloideos con células gigantes multinucleadas, algunas de tipo Langhans y necrosis central caseificante. Alrededor se reconocen linfocitos normotípicos. Los hallazgos morfológicos son compatibles con pericarditis granulomatosa caseificante secundario a infección por Mycobacterium tuberculosis. 
ción de eyección de $53 \%$ asociado a un pericardio engrosado con derrame pericárdico leve y algunos elementos de pericarditis constrictiva. Es evaluado por cardiología y se decide agregar al manejo ambulatorio aspirina en dosis antiinflamatorias (500 mg cada $8 \mathrm{~h}$ ) asociado a colchicina $0,5 \mathrm{mg}$ día evolucionando de forma favorable y en la fase trisemanal del tratamiento antituberculoso con buena adherencia.

El paciente dio su consentimiento por escrito de manera informada y voluntaria para fines de esta publicación.

\section{Discusión}

El diagnóstico de la TBC pericárdica no es simple ${ }^{3}$, en el contexto que la tuberculosis sea endémica, el diagnóstico es a través de un score, validado por la European Society of Cardiology ${ }^{8}$ (Tabla 1). Por el contrario, en un contexto donde la enfermedad no es habitual, el diagnóstico se realizará mediante técnicas más complejas.

Desde el punto de vista clínico la TBC pericárdica puede presentarse a cualquier edad, siendo más frecuente en hombres entre la tercera y quinta década de la vida ${ }^{5,9,10}$. La presentación clínica se caracteriza por dolor torácico, tos, disnea, sudoración nocturna, ortopnea, edema y baja de peso $^{3}$, al examen físico se presenta con cardiomegalia, frote pericárdico, fiebre, taquicardia, pulso paradójico, hepatomegalia, ingurgitación yugular y derrame pleural $^{8,11}$.

La radiografía de tórax muestra un aumento de la silueta cardíaca en más de $90 \%$ de los casos, signos de TBC pulmonar activa en $30 \%$ de los casos y derrame pleural en $40-60 \%$ de los $\operatorname{casos}^{10}$. La tomografía de tórax muestra derrame y engrosamiento pericárdico, en $100 \%$ de los casos se evidencian cambios típicos en los linfonodos mediastínicos ${ }^{10,12}$.

El hallazgo ecocardiográfico de derrame con filamentos fibrinosos en el pericardio visceral es típico, pero no específico de pericarditis de origen tuberculoso ${ }^{10,13}$. El estudio citoquímico del líquido pericárdico muestra un exudado con alta concentración de proteínas y leucocitos, el cual, aunque característico, no es patognomónico de esta entidad ${ }^{14}$. La baciloscopia de líquido pericárdico tiene un rendimiento moderado, siendo positiva solamente entre 40 a $60 \%$ de los $\operatorname{casos}^{15}$. La medición de los niveles de interferón- $\gamma$ en
Tabla 1. Score diagnostico de pericarditis tuberculosa en zonas endémicas

\begin{tabular}{|lc|}
\hline Criterios & Puntaje \\
\hline Sudoración nocturna & 1 \\
Fiebre & 1 \\
Pérdida de peso & 2 \\
Nivel de Globulina $>40 \mathrm{~g} / \mathrm{L}$ & 3 \\
Leucocitos menor $10 \times 10^{3} \mathrm{~mm}^{3}$ & 3 \\
\hline
\end{tabular}

Puntaje $\geq 6$ es altamente sugerente de pericarditis tuberculosa en zonas endémicas. (Adaptado de Adler $Y$, Charron $P$, Imazio M, et al. 2015 ESC Guidelines for the diagnosis and management of pericardial diseases: The Task Force for the Diagnosis and Management of Pericardial Diseases of the European Society of Cardiology (ESC)Endorsed by: The European Association for Cardio-Thoracic Surgery (EACTS). Eur Heart J. 2015;36(42):2921-2964.

tejido pericárdico ha mostrado ser una valiosa herramienta para el diagnóstico rápido y precoz de la TBC Pericardica, con estudios que muestran una sensibilidad y especificidad de hasta $100 \%$ al usarse un valor de corte sobre $200 \mathrm{pg} / \mathrm{L}^{3}$.

El Xpert-MTB/RIF es una prueba basada en la reacción de la polimerasa en cadena (PCR). permite evaluar de forma rápida $(<2 \mathrm{~h})$ la presencia de ADN de Mycobacterium tuberculosis, además de su sensibilidad a rifampicina ${ }^{14}$. En líquido pericárdico el examen posee una sensibilidad y especificidad de $63,8 \%$ y $100 \%$, respectivamente.

La biopsia de tejido pericárdico puede ser útil para el diagnóstico con una eficacia diagnóstica de 10-64\%. Los hallazgos histológicos típicos incluyen necrosis caseosa e inflamación granulomatosa ${ }^{3}$.

El manejo farmacológico consiste en la terapia antituberculosa clásica, caracterizado por la administración de rifampicina, isoniazida, etambutol y pirazinamida en dosis diarias por 2 meses y luego rifampicina e isoniazida de forma trisemanal por 4 meses. Algunos estudios sobre en laboratorio reportan que la Isoniazida es la única droga con una penetrancia aceptable en el líquido pericárdico ${ }^{16}$.

El uso de corticoides es controversial, la IDSA en el 2016 sugiere que el uso de corticoides no se utilice de forma rutinaria, reservándose para pacientes con alto riesgo de complicaciones inflamatorias (derrame pericárdico severo, altos niveles de marcadores y/o de células inflamatorias en el líquido pericárdico y signos tempranos de pericarditis constrictiva) ${ }^{17}$. 
$\mathrm{La}$ aspirina en dosis antiinflamatorias por 1-2 semanas con posterior descenso ${ }^{8}$ ha sido útil en el tratamiento. Se ha utilizado en el manejo de la pericarditis constrictiva transitoria, en pacientes sin compromiso hemodinámico y parámetros inflamatorios elevados, con el objetivo de prevenir la necesidad de pericardiectomía ${ }^{18}$. Actualmente existen pocos estudios que evalúen el efecto de la colchicina en la pericarditis tuberculosa.

No hay estudios que comparen el drenaje pericárdico de rutina o sólo si el taponamiento es clínicamente evidente ${ }^{10}$. La pericardiectomía suele indicarse cuando el tratamiento con terapia antituberculosa no logra aliviar los síntomas o la condición clínica del paciente se agrava, tras 4 a 8 semanas posterior al inicio de su administración ${ }^{8}$.

\section{Conclusión}

Actualmente con el aumento de la población migrante y el consiguiente aumento de la prevalencia de tuberculosis nacional, el medico debe estar mas atento a formas de presentación atípicas en nuestro medio.

A pesar de que el cultivo de Koch sigue siendo el gold-standard para el diagnóstico de TBC, hoy en día existen nuevos exámenes que proporcionan resultados de forma más rápida e igual de efectivos, los cuales podrían facilitar el inicio de una terapia precoz.

El inicio precoz del tratamiento logra reducir la incidencia de pericarditis constrictiva alrededor de un 60 a $80 \%$; siendo este el objetivo principal. El uso de corticoides es controversial por lo que recomendamos seguir las directrices de la ATS/ CDC/IDSA y no indicarlos de forma rutinaria

\section{Referencias}

1. Global tuberculosis report 2019. Geneva: World Health Organization; 2019.

2. Howlett P, Du Bruyn E, Morrison H, Godsent IC, Wilkinson KA, Ntsekhe M, et al. The immunopathogenesis of tuberculous pericarditis. Microbes Infect 2020; 22(45): 172-81.

3. Chang SA. Tuberculous and Infectious Pericarditis. Cardiol Clin 2017; 35 (4): 615-22.

4. Escobar S. Nadia. Situación epidemiológica de la tuberculosis en Chile en el escenario global 2018. Rev Chil Enferm Respir 2019; 35 (1): 63-70.
5. Tapia E, Oscar. Pericarditis Constrictiva Tuberculosa: Reporte de un Caso y Revisión de la Literatura. Int J Morphol 2012; 30 (2): 696-700.

6. Lasso B, Martín, Pérez G, Jorge. Pericarditis por Mycobacterium tuberculosis multiresistente en un paciente con infección por VIH: Reporte de un caso clínico y revisión de la literatura. Rev Chilena Infectol 2009; 26 (2): 15661.

7. En las Américas, Tuberculosis. OPS-OMS Washington, DC. Documento OPS/CDE/18-036, 2018.

8. Adler Y, Charron P, Imazio M, Badano L, Barón-Esquivias G, Bogaert J, et al. 2015 ESC Guidelines for the diagnosis and management of pericardial diseases: The Task Force for the Diagnosis and Management of Pericardial Diseases of the European Society of Cardiology (ESC)Endorsed by: The European Association for Cardio-Thoracic Surgery (EACTS). Eur Heart J 2015; 36 (42): 2921-64.

9. Isiguzo G, Du Bruyn E, Howlett P, Ntsekhe M. Diagnosis and Management of Tuberculous Pericarditis: What Is New? Curr Cardiol Rep. 2020; 22 (1): 2.

10. Mayosi BM, Burgess LJ, Doubell AF. Tuberculous pericarditis. Circulation. 2005; 112 (23): 3608-16.

11. Trautner BW, Darouiche RO. Tuberculous pericarditis: optimal diagnosis and management. Clin Infect Dis. 2001; 33 (7): 954-61.

12. Cherian G. Diagnosis of tuberculous aetiology in pericardial effusions. Postgrad Med J. 2004; 80 (943): 262-6.

13. Liu PY, Li YH, Tsai WC, Tsai LM, Chao TH, Yung YJ, et al. Usefulness of echocardiographic intrapericardial abnormalities in the diagnosis of tuberculous pericardial effusion. Am J Cardiol. 2001; 87 (9): 1133-335.

14. Vallejo VP, Rodríguez DJC, Searle MA, Farga CV. Ensayo Xpert MTB/RIF en el diagnóstico de tuberculosis. Revista chilena de enfermedades respiratorias. 2015; 31 (2): 127-31.

15. Fanlo P, Tiberio G. Tuberculosis extrapulmonar. Anales Sis San Navarra 2007; 30 (2): 143-62.

16. Shenje J, Ifeoma Adimora-Nweke F, Ross IL, et al. Poor Penetration of Antibiotics Into Pericardium in Pericardial Tuberculosis. EBioMedicine. 2015; 2 (11): 1640-9.

17. Nahid P, Dorman SE, Alipanah N, et al. Executive Summary: Official American Thoracic Society/Centers for Disease Control and Prevention/Infectious Diseases Society of America Clinical Practice Guidelines: Treatment of Drug-Susceptible Tuberculosis. Clin Infect Dis. 2016; 63 (7): 853-67.

18. Syed FF, Schaff HV, Oh JK. Constrictive pericarditis-a curable diastolic heart failure [published correction appears in Nat Rev Cardiol. 2015; 12 (12): 682]. Nat Rev Cardiol. 2014; 11 (9): 530-44. 